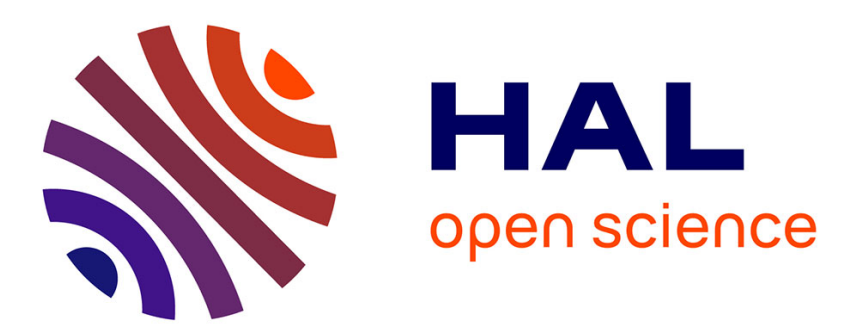

\title{
Physical systems with random uncertainties: Chaos representations with arbitrary probability measure
}

\author{
Christian Soize, R. Ghanem
}

\section{To cite this version:}

Christian Soize, R. Ghanem. Physical systems with random uncertainties: Chaos representations with arbitrary probability measure. SIAM Journal on Scientific Computing, 2004, 26 (2), pp.395-410. $10.1137 /$ S1064827503424505 . hal-00686211

\section{HAL Id: hal-00686211 https://hal.science/hal-00686211}

Submitted on 8 Apr 2012

HAL is a multi-disciplinary open access archive for the deposit and dissemination of scientific research documents, whether they are published or not. The documents may come from teaching and research institutions in France or abroad, or from public or private research centers.
L'archive ouverte pluridisciplinaire HAL, est destinée au dépôt et à la diffusion de documents scientifiques de niveau recherche, publiés ou non, émanant des établissements d'enseignement et de recherche français ou étrangers, des laboratoires publics ou privés. 


\title{
PHYSICAL SYSTEMS WITH RANDOM UNCERTAINTIES: CHAOS REPRESENTATIONS WITH ARBITRARY PROBABILITY MEASURE*
}

\author{
CHRISTIAN SOIZE $^{\dagger}$ AND ROGER GHANEM ${ }^{\ddagger}$
}

\begin{abstract}
The basic random variables on which random uncertainties can in a given model depend can be viewed as defining a measure space with respect to which the solution to the mathematical problem can be defined. This measure space is defined on a product measure associated with the collection of basic random variables. This paper clarifies the mathematical structure of this space and its relationship to the underlying spaces associated with each of the random variables. Cases of both dependent and independent basic random variables are addressed. Bases on the product space are developed that can be viewed as generalizations of the standard polynomial chaos approximation. Moreover, two numerical constructions of approximations in this space are presented along with the associated convergence analysis.
\end{abstract}

Key words. stochastic analysis, uncertainty quantification, stochastic representations

AMS subject classifications. 60H35, 60H15, 60H25, 60H40, 65C50

DOI. $10.1137 / \mathrm{S} 1064827503424505$

1. Introduction. Characterizing the membership of a mathematical function in the most suitable functional space is a critical step toward analyzing it and identifying sequences of efficient approximants to it. In most cases encountered in scientific computing, many of the relevant functional spaces are associated with the same Lebesgue measure which is often omitted from the analysis. However, in the context of modeling physical systems that exhibit uncertainty either in their behavior or in their environment, probability theory is often used as a framework for modeling the uncertainty $[14,10,20,21]$. In these cases, the functional spaces over which the various quantities of interest are defined are associated with different measures. These are typically mixtures of probability measures, each tagging the probabilistic content of some related function [3]. This paper describes the most general mathematical setting for characterizing such problems in the case where random uncertainties are defined by a finite number of basic vector-valued random variables with arbitrary probability distributions. This arbitrariness is manifested by the possibility of a multidimensional, non-Gaussian probability measure for each basic vector-valued random variable, whose components are a set of generally dependent random variables.

In the paper, a general stochastic physical system is thought of as a nonlinear transformation of a finite set of basic random variables defined over a suitable product space. For clarity of presentation, and without loss of generality, the paper deals with transformations taking values in a finite-dimensional vector space. The extension to transformations with values in a Hilbert space can be readily obtained, with the present case being a finite-dimensional approximation, such as obtained via finite

*Received by the editors March 24, 2003; accepted for publication (in revised form) March 4, 2004; published electronically December 22, 2004. The authors acknowledge the financial support of Sandia National Laboratories through contract 14652 and the Office of Naval Research under grant N000149910900.

http://www.siam.org/journals/sisc/26-2/42450.html

†Laboratoire de Mecanique, Université Marne La-Vallée, 5, Boulevard Descartes, 77454 Marnela-Vallée cedex 2, France (soize@univ-mlv.fr).

$\ddagger 201$ Latrobe Hall, The Johns Hopkins University, Baltimore, MD 21218 (ghanem@jhu.edu). 
element formalisms.

Clearly, the finite-dimensional assumption on the basic random variables corresponds to the situation where the uncertainty in the problem is inherently associated with a finite number of random variables. This assumption can also be justified for situations where the uncertainty derives from infinite-dimensional stochastic processes or fields that have been reduced through adapted techniques such as the KarhunenLoeve expansion or the polynomial chaos decomposition $[22,17,10]$.

The finite-dimensional character of the basic random variables permits the natural extension of the standard Wiener chaos decomposition, well defined for Gaussian basic random variables, to the case of second-order random variables with arbitrary probability measure. The Hilbert space to which the random solution of the mathematical problem belongs can be constructed as a tensor product of Hilbert spaces associated with the basic variables. Each of these spaces is itself written as a tensor product of Hilbert spaces.

In this paper, the finite-dimensional chaos decomposition is constructed as a Hilbertian basis of the Hilbert space of the solution, taking into consideration the tensorized structure of this vector space. This Hilbertian basis is thus obtained as the tensor product of Hilbertian bases associated with the basic random variables. This construction differs from two standard constructions. The first one deals with the case of Gaussian infinite-dimensional basic variables for which mathematical methods used for the construction of Fock spaces are applicable [12, 19, 11]. The second standard method deals with multidimensional polynomial approximations over product vector spaces. The standard approach to this problem coincides with the stochastic problem in the case where each basic random variable consists of mutually independent components. The present work, therefore, can be viewed as an extension of the second construction to relax the independence assumption.

It should be noted that the mathematical tools used in this paper consist of standard results in Hilbert spaces, specifically, the Hilbertian basis theorem and the orthogonal projection theorem [13]. It is shown that these simple tools can be used to construct a complete mathematical framework in which efficient solutions can be developed to physical systems with general random uncertainties modeled by basic vector-valued random variables which are not necessarily Gaussian, and for which the components are not necessarily independent. The resulting mathematical framework is very well suited for the analysis, within a computational context, of these systems. Chaos decomposition techniques have indeed been recently applied to a wide range of problems in scientific computing relating to uncertain systems $[8,9,7,18,6,5$, $2,16,4,23]$. Attempts at developing chaos decompositions that are adapted to nonGaussian basic variables have also been presented in the literature [23], and the present work can be viewed as delineating the correct mathematical framework in which these extensions to these efforts should be described.

The paper is self-contained in that quantities are defined when they first appear and enough detail is provided to assist the reader in implementing the framework. In the first part of the paper (sections 2-5), the mathematical construction is carried out for the finite-dimensional chaos representation for vector-valued random variables with arbitrary probability measure. In the second part (section 6), the implementation of the chaos decomposition is demonstrated through its application to the model of a physical system with random uncertainties. Construction and convergence issues are also addressed. Finally, in the third part (the appendix), data is provided for the construction of orthogonal polynomials with respect to the most common probability 
measures.

2. Defining vector-valued random variables. Consider a physical system featuring random uncertainties in some of the parameters of its mathematical model. The random uncertainties are identified with the $p$ basic vector-valued random variables $\boldsymbol{Z}^{1}, \ldots, \boldsymbol{Z}^{p}$. The solution describing the behavior of the physical system is a vector-valued random variable $\boldsymbol{Y}=\widetilde{\boldsymbol{f}}\left(\boldsymbol{Z}^{1}, \ldots, \boldsymbol{Z}^{p}\right)$ in which $\widetilde{\boldsymbol{f}}$ is a nonlinear mapping.

Consider an $\mathbb{R}^{m_{j}}$-valued random variable $\boldsymbol{Z}^{j}$ defined on a probability space $(\mathcal{A}, \mathcal{T}, P)$ with mean $\boldsymbol{m}_{Z^{j}}$ and a positive-definite covariance matrix $\boldsymbol{C}_{\boldsymbol{Z}^{j}}$ admitting the Cholesky factorization

$$
\boldsymbol{C}_{\boldsymbol{Z}^{j}}=\boldsymbol{L}_{\boldsymbol{Z}^{j}}^{T} \boldsymbol{L}_{\boldsymbol{Z}^{j}}
$$

Then, $\boldsymbol{Z}_{j}$ can be normalized:

$$
\boldsymbol{Z}^{j}=\boldsymbol{m}_{Z^{j}}+\boldsymbol{L}_{Z^{j}}^{T} \boldsymbol{X}^{j}
$$

where $\boldsymbol{X}^{j}$ is an $\mathbb{R}^{m_{j}}$-valued random variable with mean zero and covariance matrix equal to the identity. Consequently, random variable $\boldsymbol{Y}=\widetilde{\boldsymbol{f}}\left(\boldsymbol{Z}^{1}, \ldots, \boldsymbol{Z}^{p}\right)$ can be rewritten as $\boldsymbol{Y}=\boldsymbol{f}\left(\boldsymbol{X}^{1}, \ldots, \boldsymbol{X}^{p}\right)$ in which the nonlinear mapping $\boldsymbol{f}$ is such that $\boldsymbol{f}\left(\boldsymbol{X}^{1}, \ldots, \boldsymbol{X}^{p}\right)=\widetilde{\boldsymbol{f}}\left(\boldsymbol{m}_{Z^{1}}+\boldsymbol{L}_{Z^{1}}^{T} \boldsymbol{X}^{1}, \ldots, \boldsymbol{m}_{Z^{p}}+\boldsymbol{L}_{Z^{p}}^{T} \boldsymbol{X}^{p}\right)$. Thus, without any loss of generality, the subsequent analysis will be carried out for normalized random vectors. Next, consider the measurable function $\left(\boldsymbol{x}^{1}, \ldots, \boldsymbol{x}^{p}\right) \mapsto \boldsymbol{f}\left(\boldsymbol{x}^{1}, \ldots, \boldsymbol{x}^{p}\right)$ from $\mathbb{R}^{m_{1}} \times$ $\cdots \times \mathbb{R}^{m_{p}}$ into $\mathbb{C}^{m}$. Moreover, let $P_{\boldsymbol{X}^{1}, \ldots, \boldsymbol{X}^{p}}\left(d \boldsymbol{x}^{1}, \ldots, d \boldsymbol{x}^{p}\right)$ be the probability measure of the random variable $\left(\boldsymbol{X}^{1}, \ldots, \boldsymbol{X}^{p}\right)$ with values in $\mathbb{R}^{m_{1}} \times \cdots \times \mathbb{R}^{m_{p}}$ and let $\boldsymbol{Y}=$ $\boldsymbol{f}\left(\boldsymbol{X}^{1}, \ldots, \boldsymbol{X}^{p}\right)$ be a $\mathbb{C}^{m}$-valued random variable. Furthermore, assume that $\boldsymbol{Y}$ is a second-order random variable, that is,

$$
E\left\{\left\|\boldsymbol{f}\left(\boldsymbol{X}^{1}, \ldots, \boldsymbol{X}^{p}\right)\right\|^{2}\right\}<+\infty
$$

in which $E\{$.$\} denotes the mathematical expectation, and where \|$.$\| denotes the$ Hermitian norm in $\mathbb{C}^{m}$ associated with the inner product $\langle\boldsymbol{f}, \boldsymbol{g}\rangle_{\mathbb{C}^{m}}=\sum_{j=1}^{m} f_{j} \bar{g}_{j}$, in which an overbar denotes complex conjugation. This inner product reduces in an obvious manner to the real case. It is assumed that the random vectors $\boldsymbol{X}^{1}, \ldots, \boldsymbol{X}^{p}$ are mutually independent, resulting in

$$
P_{\boldsymbol{X}^{1}, \ldots, \boldsymbol{X}^{p}}=P_{\boldsymbol{X}^{1}} \otimes \cdots \otimes P_{\boldsymbol{X}^{p}},
$$

where $P_{\boldsymbol{X}^{j}}$ is the probability distribution of random variable $\boldsymbol{X}^{j}$. Let $L_{\mu}^{2}(F, G)$ denote the space of $\mu$-square-integrable functions from topological vector space $F$ into topological vector space $G$, in which $\mu$ is a probability measure on $F$ equipped with its Borel field.

3. Hilbert spaces for finite-dimensional chaos representations. A mathematical structure appropriate for the construction of the finite-dimensional chaos representation requires the characterization of the complex Hilbert space $\mathbb{H}^{(m)}$ to which $\left(\boldsymbol{x}^{1}, \ldots, \boldsymbol{x}^{p}\right) \mapsto \boldsymbol{f}\left(\boldsymbol{x}^{1}, \ldots, \boldsymbol{x}^{p}\right)$ belongs. Given the multiparameter dependence of $\boldsymbol{Y}=\boldsymbol{f}\left(\boldsymbol{X}^{1}, \ldots, \boldsymbol{X}^{p}\right)$ and the multidimensional nature of each of these parameters, real Hilbert spaces $\mathbb{H}_{j}$ and $\mathbb{H}$, associated with the measures $P_{\boldsymbol{X}^{j}}\left(d \boldsymbol{x}^{j}\right)$ and 
$P_{\boldsymbol{X}^{1}, \ldots, \boldsymbol{X}^{p}}\left(d \boldsymbol{x}^{1}, \ldots, d \boldsymbol{x}^{p}\right)$, must also be characterized:

$$
\begin{aligned}
\mathbb{H}_{j} & =L_{P_{\mathbf{X}^{j}}}^{2}\left(\mathbb{R}^{m_{j}}, \mathbb{R}\right), \\
\mathbb{H}^{(m)} & =L_{P_{\mathbf{X}^{1}, \ldots, \mathbf{X}^{p}}}^{2}\left(\mathbb{R}^{m_{1}} \times \cdots \times \mathbb{R}^{m_{p}}, \mathbb{C}^{m}\right) \\
& \simeq\left(L_{P_{\mathbf{X}^{1}, \ldots, \boldsymbol{X}^{p}}}^{2}\left(\mathbb{R}^{m_{1}} \times \cdots \times \mathbb{R}^{m_{p}}, \mathbb{R}\right)\right) \otimes \mathbb{C}^{m} \\
& =\left(\otimes_{j=1}^{p} \mathbb{H}_{j}\right) \otimes \mathbb{C}^{m} .
\end{aligned}
$$

We can then write $\mathbb{H}^{(m)}$ as

$$
\mathbb{H}^{(m)}=\mathbb{H} \otimes \mathbb{C}^{m},
$$

in which $\mathbb{H}$ is a real Hilbert space defined by

$$
\mathbb{H}=\otimes_{j=1}^{p} \mathbb{H}_{j} .
$$

Throughout this paper, the tensor product $H_{1} \otimes H_{2}$ of two real Hilbert spaces $H_{1}$ and $H_{2}$ is defined with respect to the universal property of the tensor product. In addition, in order to simplify the notation, the tensor product $H_{1} \otimes H_{2}$ has to be understood as the completion $H_{1} \widehat{\otimes} H_{2}$ of the space $H_{1} \otimes H_{2}$.

Real Hilbert space $\mathbb{H}_{j}$ and complex Hilbert space $\mathbb{H}^{(m)}$ are equipped with the following inner products:

$$
\begin{aligned}
\langle u, v\rangle_{\mathbb{H}_{j}} & =\int_{\mathbb{R}^{m_{j}}} u\left(\boldsymbol{x}^{j}\right) v\left(\boldsymbol{x}^{j}\right) P_{\boldsymbol{X}^{j}}\left(d \boldsymbol{x}^{j}\right) \\
& =E\left\{u\left(\boldsymbol{X}^{j}\right) v\left(\boldsymbol{X}^{j}\right)\right\}
\end{aligned}
$$

and

$$
\begin{aligned}
\langle\boldsymbol{f}, \boldsymbol{g}\rangle_{\mathbb{H}^{(m)}} & =\int_{\mathbb{R}^{m_{1}}} \ldots \int_{\mathbb{R}^{m_{p}}}\left\langle\boldsymbol{f}\left(\boldsymbol{x}^{1}, \ldots, \boldsymbol{x}^{p}\right), \boldsymbol{g}\left(\boldsymbol{x}^{1}, \ldots, \boldsymbol{x}^{p}\right)\right\rangle_{\mathbb{C}^{m}} P_{\boldsymbol{X}^{1}, \ldots, \boldsymbol{X}^{p}}\left(d \boldsymbol{x}^{1}, \ldots, d \boldsymbol{x}^{p}\right) \\
& =E\left\{\left\langle\boldsymbol{f}\left(\boldsymbol{X}^{1}, \ldots, \boldsymbol{X}^{p}\right), \boldsymbol{g}\left(\boldsymbol{X}^{1}, \ldots, \boldsymbol{X}^{p}\right)\right\rangle_{\mathbb{C}^{m}}\right\} .
\end{aligned}
$$

4. Finite-dimensional chaos representation. The chaos representation of random variable $\boldsymbol{Y}=\boldsymbol{f}\left(\boldsymbol{X}^{1}, \ldots, \boldsymbol{X}^{p}\right)$ is obtained by representing $\left(\boldsymbol{x}^{1}, \ldots, \boldsymbol{x}^{p}\right) \mapsto$ $\boldsymbol{f}\left(\boldsymbol{x}^{1}, \ldots, \boldsymbol{x}^{p}\right)$ on a Hilbertian basis (complete orthonormal family of functions) of $\mathbb{H}^{(m)}$. Since $\boldsymbol{X}=\left(\boldsymbol{X}^{1}, \ldots, \boldsymbol{X}^{p}\right)$ is a random variable with values in a finite-dimensional vector space, the associated chaos representation of $\boldsymbol{Y}$ is said to be finite-dimensional.

4.1. Hilbertian basis for $\mathbb{H}_{j}$. Consider a Hilbertian basis of real Hilbert space $\mathbb{H}_{j}$ given by $\left\{\phi_{\boldsymbol{\alpha}^{j}}^{j}, \boldsymbol{\alpha}^{j}=\left(\alpha_{1}^{j}, \ldots, \alpha_{m_{j}}^{j}\right) \in \mathbb{N}^{m_{j}}\right\}$; thus

$$
\begin{aligned}
\left\langle\phi_{\boldsymbol{\alpha}^{j}}^{j}, \phi_{\boldsymbol{\beta}^{j}}^{j}\right\rangle_{\mathbb{H}_{j}} & =E\left\{\phi_{\boldsymbol{\alpha}^{j}}^{j}\left(\boldsymbol{X}^{j}\right) \phi_{\boldsymbol{\beta}^{j}}^{j}\left(\boldsymbol{X}^{j}\right)\right\} \\
& =\delta_{\boldsymbol{\alpha}^{j} \boldsymbol{\beta}^{j}} .
\end{aligned}
$$

Therefore any function $h \in \mathbb{H}_{j}$ can be expanded as

$$
h\left(\boldsymbol{x}^{j}\right)=\sum_{\boldsymbol{\alpha}^{j} \in \mathbb{N}^{m_{j}}} h_{\boldsymbol{\alpha}_{j}} \phi_{\boldsymbol{\alpha}^{j}}^{j}\left(\boldsymbol{x}^{j}\right),
$$


in which

$$
\begin{aligned}
h_{\boldsymbol{\alpha}_{j}} & =\left\langle h, \phi_{\boldsymbol{\alpha}^{j}}^{j}\right\rangle_{\mathbb{H}_{j}} \\
& =E\left\{h\left(\boldsymbol{X}^{j}\right) \phi_{\boldsymbol{\alpha}^{j}}^{j}\left(\boldsymbol{X}^{j}\right)\right\} .
\end{aligned}
$$

Since the real constants belong to $\mathbb{H}_{j}$, if the Hilbertian basis is chosen such that

$$
E\left\{\phi_{\boldsymbol{\alpha}^{j}}^{j}\right\}=0 \quad \forall \boldsymbol{\alpha}^{j} \neq \mathbf{0}
$$

then

$$
\phi_{\mathbf{0}}=1
$$

It should be noted that polynomial bases in general satisfy this condition.

4.2. Hilbertian basis for $\mathbb{H}^{(\boldsymbol{m})}$. Let $\left\{\boldsymbol{b}^{1}, \ldots, \boldsymbol{b}^{m}\right\}$ be the canonical basis of $\mathbb{R}^{m}$, which is then also a basis for $\mathbb{C}^{m}$. Then a Hilbertian basis of complex Hilbert space $\mathbb{H}^{(m)}$ is given by

$$
\left\{\left(\phi_{\boldsymbol{\alpha}^{1}}^{1} \otimes \cdots \otimes \phi_{\boldsymbol{\alpha}^{p}}^{p}\right) \otimes \boldsymbol{b}^{j}, \quad \boldsymbol{\alpha}^{1} \in \mathbb{N}^{m_{1}}, \ldots, \boldsymbol{\alpha}^{p} \in \mathbb{N}^{m_{p}}, \quad j=1, \ldots, m\right\} .
$$

4.3. Representation of $\boldsymbol{f}$ in $\mathbb{H}^{(\boldsymbol{m})}$. By (3), mapping $\left(\boldsymbol{x}^{1}, \ldots, \boldsymbol{x}^{p}\right) \mapsto \boldsymbol{f}\left(\boldsymbol{x}^{1}, \ldots, \boldsymbol{x}^{p}\right)$ from $\mathbb{R}^{m_{1}} \times \cdots \times \mathbb{R}^{m_{p}}$ into $\mathbb{C}^{m}$ belongs to $\mathbb{H}^{(m)}$ and can then be written as

$$
\boldsymbol{f}\left(\boldsymbol{x}^{1}, \ldots, \boldsymbol{x}^{p}\right)=\sum_{\boldsymbol{\alpha}^{1} \in \mathbb{N}^{m_{1}}} \cdots \sum_{\boldsymbol{\alpha}^{p} \in \mathbb{N}^{m_{p}}} \sum_{j=1}^{m} f_{j, \boldsymbol{\alpha}^{1} \cdots \boldsymbol{\alpha}^{p}} \phi_{\boldsymbol{\alpha}^{1}}^{1}\left(\boldsymbol{x}^{1}\right) \times \cdots \times \phi_{\boldsymbol{\alpha}^{p}}^{p}\left(\boldsymbol{x}^{p}\right) \boldsymbol{b}^{j},
$$

in which

$$
\begin{aligned}
& f_{j, \boldsymbol{\alpha}^{1} \cdots \boldsymbol{\alpha}^{p}}=\left\langle\boldsymbol{f},\left(\phi_{\boldsymbol{\alpha}^{1}}^{1} \otimes \cdots \otimes \phi_{\boldsymbol{\alpha}^{p}}^{p}\right) \otimes \boldsymbol{b}^{j}\right\rangle_{\mathbb{H}^{(m)}} \\
& =\int_{\mathbb{R}^{m_{1}}} \cdots \int_{\mathbb{R}^{m_{p}}}\left\langle\boldsymbol{f}\left(\boldsymbol{x}^{1}, \ldots, \boldsymbol{x}^{p}\right), \boldsymbol{b}^{j}\right\rangle_{\mathbb{C}^{m}} \\
& \times \phi_{\boldsymbol{\alpha}^{1}}^{1}\left(\boldsymbol{x}^{1}\right) \times \cdots \times \phi_{\boldsymbol{\alpha}^{p}}^{p}\left(\boldsymbol{x}^{p}\right) P_{\boldsymbol{X}^{1}, \ldots, \boldsymbol{X}^{p}}\left(d \boldsymbol{x}^{1}, \ldots, d \boldsymbol{x}^{p}\right) .
\end{aligned}
$$

Let

$$
f_{j}\left(\boldsymbol{x}^{1}, \ldots, \boldsymbol{x}^{p}\right)=\left\langle\boldsymbol{f}\left(\boldsymbol{x}^{1}, \ldots, \boldsymbol{x}^{p}\right), \boldsymbol{b}^{j}\right\rangle_{\mathbb{C}^{m}}
$$

denote the projection of $\boldsymbol{f}\left(\boldsymbol{x}^{1}, \ldots, \boldsymbol{x}^{p}\right)$ on $\boldsymbol{b}^{j}$. Therefore (17) can be rewritten as

$$
\boldsymbol{f}\left(\boldsymbol{x}^{1}, \ldots, \boldsymbol{x}^{p}\right)=\sum_{\boldsymbol{\alpha}^{1} \in \mathbb{N}^{m_{1}}} \cdots \sum_{\boldsymbol{\alpha}^{p} \in \mathbb{N}^{m_{p}}} \boldsymbol{f}_{\boldsymbol{\alpha}^{1} \cdots \boldsymbol{\alpha}^{p}} \phi_{\boldsymbol{\alpha}^{1}}^{1}\left(\boldsymbol{x}^{1}\right) \times \cdots \times \phi_{\boldsymbol{\alpha}^{p}}^{p}\left(\boldsymbol{x}^{p}\right),
$$

where

$$
\begin{aligned}
\boldsymbol{f}_{\boldsymbol{\alpha}^{1} \ldots \boldsymbol{\alpha}^{p}}= & \left\langle\boldsymbol{f}, \phi_{\boldsymbol{\alpha}^{1}}^{1} \otimes \cdots \otimes \phi_{\boldsymbol{\alpha}^{p}}^{p}\right\rangle_{\mathbb{H}(m) \times \mathbb{H}} \\
= & \int_{\mathbb{R}^{m_{1}}} \quad \cdots \int_{\mathbb{R}^{m_{p}}} \boldsymbol{f}\left(\boldsymbol{x}^{1}, \ldots, \boldsymbol{x}^{p}\right) \\
& \quad \times \phi_{\boldsymbol{\alpha}^{1}}^{1}\left(\boldsymbol{x}^{1}\right) \times \cdots \times \phi_{\boldsymbol{\alpha}^{p}}^{p}\left(\boldsymbol{x}^{p}\right) \quad P_{\boldsymbol{X}^{1}, \ldots, \boldsymbol{X}^{p}}\left(d \boldsymbol{x}^{1}, \ldots, d \boldsymbol{x}^{p}\right), \\
= & E\left\{\boldsymbol{f}\left(\boldsymbol{X}^{1}, \ldots, \boldsymbol{X}^{p}\right) \phi_{\boldsymbol{\alpha}^{1}}^{1}\left(\boldsymbol{X}^{1}\right) \times \cdots \times \phi_{\boldsymbol{\alpha}^{p}}^{p}\left(\boldsymbol{X}^{p}\right)\right\}
\end{aligned}
$$

and where $\langle., .\rangle_{\mathbb{H}(m)} \times \mathbb{H}$ is the bilinear form on $\mathbb{H}^{(m)} \times \mathbb{H}$ defined by the right-hand side of the second equation (21). 
4.4. Chaos representation of random variable $Y$. Consequently, the chaos representation of random variable $\boldsymbol{Y}=\boldsymbol{f}\left(\boldsymbol{X}^{1}, \ldots, \boldsymbol{X}^{p}\right)$ is

$$
\boldsymbol{Y}=\sum_{\boldsymbol{\alpha}^{1} \in \mathbb{N}^{m_{1}}} \cdots \sum_{\boldsymbol{\alpha}^{p} \in \mathbb{N}^{m_{p}}} \boldsymbol{f}_{\boldsymbol{\alpha}^{1 \cdots \boldsymbol{\alpha}^{p}}} \phi_{\boldsymbol{\alpha}^{1}}^{1}\left(\boldsymbol{X}^{1}\right) \times \cdots \times \phi_{\boldsymbol{\alpha}^{p}}^{p}\left(\boldsymbol{X}^{p}\right) .
$$

5. Construction of a Hilbertian basis for $\mathbb{H}_{\boldsymbol{j}}$. For a fixed $j$, the $\mathbb{R}^{m_{j}}$-valued random normalized variable $\boldsymbol{X}^{j}$ has probability distribution $P_{\boldsymbol{X}^{j}}\left(d \boldsymbol{x}^{j}\right)$ which is represented by a probability density function $p_{\boldsymbol{X}^{j}}\left(\boldsymbol{x}^{j}\right)$ with respect to the Lebesgue measure $d \boldsymbol{x}^{j}$. While in general the $m_{j}$ components of $\boldsymbol{X}^{j}$ are mutually dependent, the case of independent components is of particular interest. Therefore, after treating the general case, the case of mutually independent components is treated separately.

5.1. Mutually dependent components of $\boldsymbol{X}^{j}$. Let $\boldsymbol{X}^{j}=\left(X_{1}^{j}, \ldots, X_{m_{j}}^{j}\right)$ be the $\mathbb{R}^{m_{j}}$-valued random variable with probability distribution

$$
P_{\boldsymbol{X}^{j}}\left(d \boldsymbol{x}^{j}\right)=p_{\boldsymbol{X}^{j}}\left(\boldsymbol{x}^{j}\right) d \boldsymbol{x}^{j}
$$

Let $p_{X_{1}^{j}}\left(x_{1}^{j}\right), \ldots, p_{X_{m_{j}}^{j}}\left(x_{m_{j}}^{j}\right)$ be the marginal probability density functions of order 1 given by

$$
p_{X_{k}^{j}}\left(x_{k}^{j}\right)=\int_{\mathbb{R}^{m_{j}-1}} p_{\boldsymbol{X}^{j}}\left(x_{1}^{j}, \ldots, x_{k-1}^{j}, x_{k}^{j}, x_{k+1}^{j}, \ldots, x_{m_{j}}^{j}\right) d x_{1}^{j} \cdots d x_{k-1}^{j} d x_{k+1}^{j} \cdots d x_{m_{j}}^{j} .
$$

The support $S_{m_{j}}$ of $p_{\boldsymbol{X}^{j}}$ is such that

$$
p_{\boldsymbol{X}^{j}}\left(\boldsymbol{x}^{j}\right)=0 \quad \text { if } \boldsymbol{x}^{j} \notin S_{m_{j}} .
$$

It should be noted that $S_{m_{j}}$ can be $\mathbb{R}^{m_{j}}$ or any bounded or compact subset thereof. For later use, the support $s_{k}$ of density $p_{X_{k}^{j}}\left(x_{k}^{j}\right)$ is such that

$$
p_{X_{k}^{j}}\left(x_{k}^{j}\right)=0 \text { if } x_{k}^{j} \notin s_{k} .
$$

It is noted here also that $s_{k}$ can be $\mathbb{R}$ or any bounded or compact subset thereof, and that, in general, $S_{m_{j}} \neq s_{1} \times \cdots \times s_{m_{j}}$.

5.1.1. Hilbert spaces $\mathbb{H}_{\boldsymbol{j}, \boldsymbol{k}}$ and $\mathbb{K}_{\boldsymbol{j}}$. The real Hilbert space associated with marginal distribution $p_{X_{k}^{j}}\left(x_{k}^{j}\right) d x_{k}^{j}$ is now introduced. Let

$$
\mathbb{H}_{j, k}=L_{P_{X_{k}^{j}}}^{2}(\mathbb{R}, \mathbb{R})
$$

be the Hilbert space associated with the probability measure $P_{X_{k}^{j}}\left(d x_{k}^{j}\right)=p_{X_{k}^{j}}\left(x_{k}^{j}\right) d x_{k}^{j}$, equipped with the inner product

$$
\langle r, s\rangle_{\mathbb{H}_{j, k}}=\int_{\mathbb{R}} r(q) s(q) p_{X_{k}^{j}}(q) d q=E\left\{r\left(X_{k}^{j}\right) s\left(X_{k}^{j}\right)\right\}
$$

Let $\left\{\psi_{\ell}^{k}, \ell \in \mathbb{N}\right\}$ be a Hilbertian basis of real Hilbert space $\mathbb{H}_{j, k}$ which is such that

$$
\left\langle\psi_{\ell}^{k}, \psi_{\ell^{\prime}}^{k}\right\rangle_{\mathbb{H}_{j, k}}=\delta_{\ell \ell^{\prime}}
$$


Let $\mathbb{K}_{j}$ be the real Hilbert space defined by

$$
\mathbb{K}_{j}=\otimes_{k=1}^{m_{j}} \mathbb{H}_{j, k}
$$

equipped with the inner product

$$
\begin{aligned}
\langle u, v\rangle_{\mathbb{K}_{j}}= & \int_{\mathbb{R}} \cdots \int_{\mathbb{R}} u\left(q_{1}, \ldots, q_{m_{j}}\right) v\left(q_{1}, \ldots, q_{m_{j}}\right) \\
& \times p_{X_{1}^{j}}\left(q_{1}\right) \times \cdots \times p_{X_{m_{j}}^{j}}\left(q_{m_{j}}\right) d q_{1} \cdots d q_{m_{j}} .
\end{aligned}
$$

It should be noted that $\langle u, v\rangle_{\mathbb{K}_{j}}$ is different from $E\left\{u\left(\boldsymbol{X}^{j}\right) v\left(\boldsymbol{X}^{j}\right)\right\}$ which is equal to $\langle u, v\rangle_{\mathbb{H}_{j}}$. From (30), the following Hilbertian basis for $\mathbb{K}_{j}$ is deduced:

$$
\left\{\psi_{\ell_{1}}^{1} \otimes \cdots \otimes \psi_{\ell_{m_{j}}}^{m_{j}}, \quad \ell_{1} \in \mathbb{N}, \ldots, \ell_{m_{j}} \in \mathbb{N}\right\}
$$

\subsubsection{Hilbertian basis for $\mathbb{H}_{\boldsymbol{j}}$.}

Lemma 1. For all $\boldsymbol{x}^{j}=\left(x_{1}^{j}, \ldots, x_{m_{j}}^{j}\right)$ belonging to the support $S_{m_{j}}$ of $p_{\boldsymbol{X}^{j}}\left(\boldsymbol{x}^{j}\right)$, Hilbertian basis $\left\{\phi_{\boldsymbol{\alpha}^{j}}^{j}, \quad \boldsymbol{\alpha}^{j} \in \mathbb{N}^{m_{j}}\right\}$ of real Hilbert space $\mathbb{H}_{j}$ is given by

$$
\phi_{\boldsymbol{\alpha}^{j}}^{j}\left(\boldsymbol{x}^{j}\right)=\left(\frac{p_{X_{1}^{j}}\left(x_{1}^{j}\right) \times \cdots \times p_{X_{m_{j}}^{j}}\left(x_{m_{j}}^{j}\right)}{p_{\boldsymbol{X}^{j}}\left(\boldsymbol{x}^{j}\right)}\right)^{1 / 2} \psi_{\alpha_{1}^{j}}^{1}\left(x_{1}^{j}\right) \times \cdots \times \psi_{\alpha_{m_{j}}^{j}}^{m_{j}}\left(x_{m_{j}}^{j}\right),
$$

where $\left\{\psi_{\alpha_{k}^{j}}^{k}\left(x_{k}^{j}\right)\right\}_{\alpha_{k}^{j}}$ is a Hilbertian basis of real Hilbert space $\mathbb{H}_{j, k}$ and $\boldsymbol{\alpha}^{j}=\left(\alpha_{1}^{j}, \ldots, \alpha_{m_{j}}^{j}\right)$.

Proof. In a first step, (33) is established. Let $h \in \mathbb{H}_{j}$, and let $\boldsymbol{x}^{j} \mapsto g\left(\boldsymbol{x}^{j}\right)$ be the function such that

$$
g\left(\boldsymbol{x}^{j}\right)=h\left(\boldsymbol{x}^{j}\right)\left(\frac{p_{\boldsymbol{X}^{j}}\left(\boldsymbol{x}^{j}\right)}{p_{X_{1}^{j}}\left(x_{1}^{j}\right) \times \cdots \times p_{X_{m_{j}}^{j}}\left(x_{m_{j}}^{j}\right)}\right)^{1 / 2}
$$

¿From (31), we deduce that $\|g\|_{\mathbb{K}_{j}}^{2}=\|h\|_{\mathbb{H}_{j}}^{2}$ and since $h \in \mathbb{H}_{j}$, we deduce that $g \in \mathbb{K}_{j}$. Therefore, this function can be expanded as

$$
\begin{gathered}
h\left(\boldsymbol{x}^{j}\right)\left(\frac{p_{\boldsymbol{X}^{j}}\left(\boldsymbol{x}^{j}\right)}{p_{X_{1}^{j}}\left(x_{1}^{j}\right) \times \cdots \times p_{X_{m_{j}}^{j}}\left(x_{m_{j}}^{j}\right)}\right)^{1 / 2}=\sum_{\alpha_{1}^{j} \in \mathbb{N}} \cdots \sum_{\alpha_{m_{j}}^{j} \in \mathbb{N}} h_{\alpha_{1}^{j} \cdots \alpha_{m_{j}}^{j}} \psi_{\alpha_{1}^{j}}^{1}\left(x_{1}^{j}\right) \\
\times \cdots \times \psi_{\alpha_{m_{j}}^{j}}^{m_{j}}\left(x_{m_{j}}^{j}\right) .
\end{gathered}
$$

Equation (34) can be rewritten as

$$
\begin{aligned}
h\left(\boldsymbol{x}^{j}\right)= & \sum_{\alpha_{1}^{j} \in \mathbb{N}} \\
& \cdots \sum_{\alpha_{m_{j}}^{j} \in \mathbb{N}} h_{\alpha_{1}^{j} \cdots \alpha_{m_{j}}^{j}}\left(\frac{p_{X_{1}^{j}}\left(x_{1}^{j}\right) \times \cdots \times p_{X_{m_{j}}^{j}}\left(x_{m_{j}}^{j}\right)}{p_{\boldsymbol{X}^{j}}\left(\boldsymbol{x}^{j}\right)}\right)^{1 / 2} \\
& \times \psi_{\alpha_{1}^{j}}^{1}\left(x_{1}^{j}\right) \times \cdots \times \psi_{\alpha_{m_{j}}^{j}}^{m_{j}}\left(x_{m_{j}}^{j}\right) .
\end{aligned}
$$

Since $h$ belongs to $\mathbb{H}_{j}$, use of (12) yields (33). 
The second step in the proof consists in verifying that the family defined by (33) is an orthonormal system in $\mathbb{H}_{j}$. A simple calculation yields the following equation:

$$
\left\langle\phi_{\boldsymbol{\alpha}^{j}}^{j}, \phi_{\boldsymbol{\beta}^{j}}^{j}\right\rangle_{\mathbb{H}_{j}}=\prod_{k=1}^{m_{j}}\left\{\left\langle\psi_{\alpha_{k}^{j}}^{k}, \psi_{\beta_{k}^{j}}^{k}\right\rangle_{\mathbb{H}_{j, k}}\right\} \text {. }
$$

Using (29) results in

$$
\left\langle\phi_{\boldsymbol{\alpha}^{j}}^{j}, \phi_{\boldsymbol{\beta}^{j}}^{j}\right\rangle_{\mathbb{H}_{j}}=\prod_{k=1}^{m_{j}} \delta_{\alpha_{k}^{j} \beta_{k}^{j}}=\delta_{\boldsymbol{\alpha}^{j} \boldsymbol{\beta}^{j}} .
$$

The final step in the proof consists in verifying that the family of functions defined by (33) is complete in $\mathbb{H}_{j}$, which is straightforward considering the completeness of Hilbertian basis $\psi_{\boldsymbol{\alpha}^{j}}^{j}$ in $\mathbb{H}_{j, k}$.

5.2. Mutually independent components of $\boldsymbol{X}^{j}$. Under the assumption of independence of the components of $\mathbb{R}^{m_{j}}$-valued random variables $\boldsymbol{X}^{j}$, the following equation holds:

$$
p_{\boldsymbol{X}^{j}}\left(\boldsymbol{x}^{j}\right)=p_{X_{1}^{j}}\left(x_{1}^{j}\right) \times \cdots \times p_{X_{m_{j}}^{j}}\left(x_{m_{j}}^{j}\right) .
$$

¿From Lemma 1, it is deduced that the Hilbertian basis $\left\{\phi_{\boldsymbol{\alpha}^{j}}^{j}, \quad \boldsymbol{\alpha}^{j} \in \mathbb{N}^{m_{j}}\right\}$ of the real Hilbert space $\mathbb{H}_{j}$ is given by

$$
\phi_{\boldsymbol{\alpha}^{j}}^{j}\left(\boldsymbol{x}^{j}\right)=\psi_{\alpha_{1}^{j}}^{1}\left(x_{1}^{j}\right) \times \cdots \times \psi_{\alpha_{m_{j}}^{j}}^{m_{j}}\left(x_{m_{j}}^{j}\right),
$$

where $\boldsymbol{x}^{j}=\left(x_{1}^{j}, \ldots, x_{m_{j}}^{j}\right)$ and $\boldsymbol{\alpha}^{j}=\left(\alpha_{1}^{j}, \ldots, \alpha_{m_{j}}^{j}\right)$. In this case, it should be noted that real Hilbert space $\mathbb{K}_{j}$ coincides with real Hilbert space $\mathbb{H}_{j}$. Moreover, it should noted in this case that the support $S_{m_{j}}$ becomes the product of the supports $s_{1}, \ldots, s_{m_{j}}$,

$$
S_{m_{j}}=s_{1} \times \cdots \times s_{m_{j}} .
$$

5.3. Classical orthogonal polynomials as bases for $\mathbb{H}_{\boldsymbol{j}, \boldsymbol{k}}$. A close connection exists between classical orthogonal polynomials and chaos decompositions. Indeed, to each of the classical polynomials is associated a weight function that can be construed as a density of a measure on an appropriate space. In the terminology used in this paper, the orthogonal polynomials refer to the Hilbertian basis $\left\{\psi_{\alpha_{k}^{j}}^{k}\left(x_{k}^{j}\right)\right\}$ of the real Hilbert space $\mathbb{H}_{j, k}$ associated with the probability measure $P_{X_{k}^{j}}\left(d x_{k}^{j}\right)=p_{X_{k}^{j}}\left(x_{k}^{j}\right) d x_{k}^{j}$ (see sections 5.1.1 and 5.1.2). For notational convenience, $\left\{\psi_{\alpha_{k}^{j}}^{k}\right\}$ is rewritten as $\psi_{\ell}(q), p_{X_{k}^{j}}\left(x_{k}^{j}\right)$ as $w(q)$, and the support $s_{k}$ is denoted by $s$. With this notation, the inner product in $\mathbb{H}_{j, k}$ is rewritten as

$$
\begin{aligned}
\left\langle\psi_{\ell}, \psi_{\ell^{\prime}}\right\rangle_{\mathbb{H}_{j k}} & =\int_{\mathbb{R}} \psi_{\ell}(q) \psi_{\ell^{\prime}}(q) w(q) d q \\
& =\int_{s} \psi_{\ell}(q) \psi_{\ell^{\prime}}(q) w(q) d q \\
& =\delta_{\ell \ell^{\prime}} .
\end{aligned}
$$

Table 1 (see appendix) shows the weights $w(q)$, the support, and the expressions of $\psi_{\ell}(q)$ for a number of classical orthogonal polynomials. Recurrence expressions for the construction of the orthogonal polynomials are given in Table 2 . 
6. Physical system with random uncertainties. This section demonstrates the implementation of the chaos decomposition through an example from dynamics.

6.1. Definition of the physical system. Consider a physical system modeled as an elliptic linear boundary-value problem over a bounded domain $B$ of $\mathbb{R}^{d}$, with $d$ a finite positive integer. Let the associated operator depend on $p_{1}$ stochastic processes, $\boldsymbol{W}^{1}(\boldsymbol{\zeta}), \ldots, \boldsymbol{W}^{p_{1}}(\boldsymbol{\zeta})$, and $p_{2}$ vector-valued random variables $\left(p=p_{1}+p_{2}\right)$, representing the uncertain behavior of the underlying physical system. Each of the $p_{1}$ random processes can itself be discretized in terms of a countable set of random variables via the Karhunen-Loeve expansion [17]. The number of terms retained in this expansion for each process depends on the correlation length of its random fluctuations, increasing as this length decreases. Each of the processes $\boldsymbol{W}^{j}(\boldsymbol{\zeta})$ is thus replaced by an $\mathbb{R}^{m_{j}}$-valued random variable $\boldsymbol{Z}^{j}$, where $m_{j}$ is the number of terms retained in the Karhunen-Loeve expansion of the $j$ th process. The operator associated with the boundary-value problem can thus be expressed as explicitly dependent on $p$ random variables $\boldsymbol{Z}^{j}$, which can be transformed into a set of orthogonal random variables $\boldsymbol{X}$ as described in (2).

6.2. Joint probability density function of the Karhunen-Loeve random variables. The representation of a stochastic process through the Karhunen-Loeve expansion results in a countable set of random variables the probability measure of which is a function of the probability measure of the process. In the case where the latter is a Gaussian process, the random variables form an orthonormal Gaussian sequence. The theoretical development presented in this paper relies on the availability of the joint probability distribution of the basic random variables on which the solution, being represented in its chaos decomposition, depends. In this section, the joint probability density function of the Karhunen-Loeve random variables is developed in terms of the probability measure of the associated process, thus permitting the integration of the Karhunen-Loeve representation into the foregoing analysis.

Let $\{\boldsymbol{Z}(\boldsymbol{\zeta}), \boldsymbol{\zeta} \in B\}$ be a second-order centered stochastic process indexed by a bounded set $B$ of $\mathbb{R}^{d}$ with values in $\mathbb{R}^{\nu}$. The matrix-valued correlation function of $\boldsymbol{Z}$ is thus given by

$$
\boldsymbol{R}_{\boldsymbol{Z}}\left(\boldsymbol{\zeta}, \boldsymbol{\zeta}^{\prime}\right)=E\left\{\boldsymbol{Z}(\boldsymbol{\zeta}) \boldsymbol{Z}\left(\boldsymbol{\zeta}^{\prime}\right)^{T}\right\}
$$

and is assumed to be such that

$$
\int_{B} \int_{B}\left\|\boldsymbol{R}_{\boldsymbol{Z}}\left(\boldsymbol{\zeta}, \boldsymbol{\zeta}^{\prime}\right)\right\|_{H S}^{2} d \boldsymbol{\zeta}^{\prime} d \boldsymbol{\zeta}<\infty
$$

where $\|\cdot\|_{H S}$ is the Hilbert-Schmidt norm. For instance, since $B$ is a bounded set of $\mathbb{R}^{d}$, the condition defined by (43) is satisfied if $\boldsymbol{Z}$ is a mean-square continuous stochastic process on $B$. Then let $\mathbb{V}=L_{d \zeta}^{2}\left(B, \mathbb{R}^{\nu}\right)$ be the Hilbert space of $\mathbb{R}^{\nu}$-valued square-integrable functions on $B$ equipped with the inner product

$$
\langle\boldsymbol{u}, \boldsymbol{v}\rangle_{\mathbb{V}}=\int_{B}\langle\boldsymbol{u}(\boldsymbol{\zeta}), \boldsymbol{v}(\boldsymbol{\zeta})\rangle_{\mathbb{R}^{\nu}} d \boldsymbol{\zeta}
$$

Due to (43), the linear operator $\boldsymbol{R}_{\boldsymbol{Z}}$ defined by the following bilinear form on $\mathbb{V} \times \mathbb{V}$,

$$
\left\langle\boldsymbol{R}_{\boldsymbol{Z}} \boldsymbol{u}, \boldsymbol{v}\right\rangle_{\mathbb{V}}=\int_{B} \int_{B}\left\langle\boldsymbol{R}_{\boldsymbol{Z}}\left(\boldsymbol{\zeta}, \boldsymbol{\zeta}^{\prime}\right) \boldsymbol{u}\left(\boldsymbol{\zeta}^{\prime}\right), \boldsymbol{v}(\boldsymbol{\zeta})\right\rangle_{\mathbb{R}^{\nu}} d \boldsymbol{\zeta}^{\prime} d \boldsymbol{\zeta}
$$


is a Hilbert-Schmidt operator [13]. Consequently, the eigenvalue problem

$$
\boldsymbol{R}_{Z} \phi=\lambda \phi
$$

has a sequence of real positive eigenvalues $\lambda_{1} \geq \lambda_{2} \geq \cdots \rightarrow 0$ such that $\sum_{j=1}^{\infty} \lambda_{j}^{2}<$ $\infty$. The associated eigenfunctions form a Hilbertian basis of $\mathbb{V}$. In this case, the Karhunen-Loeve decomposition of stochastic process $(\boldsymbol{Z}(\boldsymbol{\zeta}), \boldsymbol{\zeta} \in B)$ can be written as

$$
\boldsymbol{Z}(\boldsymbol{\zeta})=\sum_{j=1}^{\infty} \sqrt{\lambda}_{j} \xi_{j} \boldsymbol{\phi}_{j}(\boldsymbol{\zeta})
$$

with

$$
\xi_{j}=\frac{1}{\sqrt{\lambda_{j}}}\langle\boldsymbol{Z}, \boldsymbol{\phi}\rangle_{\mathbb{V}}
$$

where the sequence of random variables $\left\{\xi_{j}\right\}$ is such that

$$
\begin{gathered}
E\left\{\xi_{j}\right\}=0, \\
E\left\{\xi_{i} \xi_{j}\right\}=\delta_{i j} .
\end{gathered}
$$

Truncating the KL expansion at the $\mu$ th term defines the approximation $\boldsymbol{Z}^{\mu}(\boldsymbol{\zeta})$ as

$$
\boldsymbol{Z}^{\mu}(\zeta)=\sum_{j=1}^{\mu} \sqrt{\lambda}_{j} \xi_{j} \phi_{j}(\boldsymbol{\zeta})
$$

It is noted that

$$
E\left\{\left\|\boldsymbol{Z}-\boldsymbol{Z}^{\mu}\right\|_{\mathbb{V}}^{2}\right\}=\sum_{\mu+1}^{\infty} \lambda_{j} .
$$

For all $\boldsymbol{\theta}^{\mu}=\left(\theta_{1}^{\mu}, \ldots, \theta_{\mu}^{\mu}\right)$ in $\mathbb{R}^{\mu}$, the characteristic function of the $\mathbb{R}^{\mu}$-valued random variable $\boldsymbol{\xi}^{\mu}=\left(\xi_{1}^{\mu}, \ldots, \xi_{\mu}^{\mu}\right)$ is defined by

$$
\Phi_{\boldsymbol{\xi}^{\mu}}\left(\boldsymbol{\theta}^{\mu}\right)=E\left\{\exp \left\{i\left\langle\boldsymbol{\xi}^{\mu}, \boldsymbol{\theta}^{\mu}\right\rangle_{\mathbb{R}^{\mu}}\right\}\right\}
$$

and can be rewritten as

$$
\begin{aligned}
\Phi_{\boldsymbol{\xi}^{\mu}}\left(\boldsymbol{\theta}^{\mu}\right) & =E\left\{\exp \left(i \sum_{j=1}^{\mu} \frac{\theta_{j}^{\mu}}{\sqrt{\lambda_{j}}}\left\langle\boldsymbol{Z}, \boldsymbol{\phi}_{j}\right\rangle_{\mathbb{V}}\right)\right\} \\
& =E\left\{\exp \left(i\left\langle\boldsymbol{Z}, \boldsymbol{v}^{\mu}\right\rangle_{\mathbb{V}}\right)\right\}
\end{aligned}
$$

where

$$
\boldsymbol{v}^{\mu}(\boldsymbol{\zeta})=\sum_{j=1}^{\mu} \frac{\theta_{j}^{\mu}}{\sqrt{\lambda_{j}}} \phi_{j}(\boldsymbol{\zeta}) .
$$

Introducing the characteristic functional, $\Phi_{\boldsymbol{Z}}(\boldsymbol{v})$ of stochastic process $\boldsymbol{Z}$, defined for suitable function $\boldsymbol{v}$, the following equation results:

$$
\begin{aligned}
\Phi_{\boldsymbol{\xi}^{\mu}}\left(\boldsymbol{\theta}^{\mu}\right) & =E\left\{\exp \left(i\left\langle\boldsymbol{Z}, \boldsymbol{v}^{\mu}\right\rangle_{\mathbb{V}}\right)\right\} \\
& =\Phi_{\boldsymbol{Z}}\left(\boldsymbol{v}^{\mu}\right) .
\end{aligned}
$$


Thus given the characteristic functional of stochastic process $\boldsymbol{Z}$ and the eigenfunctions of its correlation operator, (54) and (55) can be used to obtain the characteristic function of $\boldsymbol{\xi}^{\mu}$, its truncated Karhunen-Loeve representation.

In this paper, the Karhunen-Loeve expansion is used as a means to project stochastic processes onto finite-dimensional representations. While no convergence analysis regarding the effect of these finite representations on the accuracy of the chaos decompositions is carried out, existing results [15] present an equivalence, as the number of terms in the Karhunen-Loeve expansion increases, between convergence with respect to various norms, as well as between convergence with respect to the underlying measure.

6.3. Construction of the chaos representation of the random solution and convergence analysis. The projection of the weak formulation of the elliptic boundary value problem with random uncertainties onto an $m$-dimensional subspace can be achieved through any one of a number of procedures, such as the finite element method (FEM), resulting in a random linear algebraic problem of the form

$$
\boldsymbol{A}\left(\boldsymbol{X}^{1}, \ldots, \boldsymbol{X}^{p}\right) \boldsymbol{Y}=\boldsymbol{F},
$$

where $\boldsymbol{F}$ is a given element of $\mathbb{C}^{m}$, and $\boldsymbol{A}$ is a random $m \times m$ complex matrix depending on the basic vector-valued random variables $\boldsymbol{X}^{1}, \ldots, \boldsymbol{X}^{p}$. It is moreover assumed that the $\mathbb{C}^{m}$-valued solution $\boldsymbol{Y}$ of (56) exists almost surely. Consequently, random matrix $\boldsymbol{A}\left(\boldsymbol{X}^{1}, \ldots, \boldsymbol{X}^{p}\right)^{-1}$ exists almost surely, defining a nonlinear mapping $\boldsymbol{f}$ such that

$$
\boldsymbol{Y}=\boldsymbol{f}\left(\boldsymbol{X}^{1}, \ldots, \boldsymbol{X}^{p}\right) .
$$

Finally, it is also assumed that mapping $\boldsymbol{f}$ is such that (3) is verified, and, consequently, $\boldsymbol{Y}=\boldsymbol{f}\left(\boldsymbol{X}^{1}, \ldots, \boldsymbol{X}^{p}\right)$ is the unique second-order random solution of random matrix (56).

Taking into account (22), a representation of the solution is sought with respect to the chaos basis of the form

$$
\boldsymbol{Y}=\sum_{\boldsymbol{\alpha}^{1} \in \mathbb{N}^{m_{1}}} \cdots \sum_{\boldsymbol{\alpha}^{p} \in \mathbb{N}^{m_{p}}} \boldsymbol{Y}_{\boldsymbol{\alpha}^{1 \cdots} \boldsymbol{\alpha}^{p}} \phi_{\boldsymbol{\alpha}^{1}}^{1}\left(\boldsymbol{X}^{1}\right) \times \cdots \times \phi_{\boldsymbol{\alpha}^{p}}^{p}\left(\boldsymbol{X}^{p}\right),
$$

with the $\mathbb{C}^{m}$-valued coefficient $\boldsymbol{Y}_{\boldsymbol{\alpha}^{1} \ldots \boldsymbol{\alpha}^{p}}$. With $r_{1}, \ldots, r_{p}$ denoting the lengths $\left|\boldsymbol{\alpha}^{1}\right|, \ldots$, $\left|\boldsymbol{\alpha}^{p}\right|$ of multi-indices $\boldsymbol{\alpha}_{1}, \ldots, \boldsymbol{\alpha}_{p}$, respectively, let

$$
\boldsymbol{Y}^{\boldsymbol{r}}=\sum_{\boldsymbol{\alpha}^{1},\left|\boldsymbol{\alpha}^{1}\right| \leq r_{1}} \cdots \sum_{\boldsymbol{\alpha}^{p},\left|\boldsymbol{\alpha}^{p}\right| \leq r_{p}} \boldsymbol{Y}_{\boldsymbol{\alpha}^{1 \cdots} \boldsymbol{\alpha}^{p}} \phi_{\boldsymbol{\alpha}^{1}}^{1}\left(\boldsymbol{X}^{1}\right) \times \cdots \times \phi_{\boldsymbol{\alpha}^{p}}^{p}\left(\boldsymbol{X}^{p}\right),
$$

where $\left|\alpha^{k}\right|=\sum_{j=1}^{m_{k}} \alpha_{j}^{k}$ and $\boldsymbol{r}=\left(r_{1}, \ldots, r_{p}\right)$.

6.3.1. Construction through sampling and convergence analysis. According to the third equation in (21), coefficients $\boldsymbol{Y}_{\boldsymbol{\alpha}^{1 \ldots \boldsymbol{\alpha}^{p}}}$ of the chaos development of $\boldsymbol{Y}$ are given by the following expression:

$$
\boldsymbol{Y}_{\boldsymbol{\alpha}^{1 \cdots} \boldsymbol{\alpha}^{p}}=E\left\{\boldsymbol{Y} \phi_{\boldsymbol{\alpha}^{1}}^{1}\left(\boldsymbol{X}^{1}\right) \times \cdots \times \phi_{\boldsymbol{\alpha}^{p}}^{p}\left(\boldsymbol{X}^{p}\right)\right\} .
$$

Equation (60) is well adapted for the evaluation of the coefficients $\boldsymbol{Y}_{\boldsymbol{\alpha}^{1 \ldots}} \boldsymbol{\alpha}^{p}$ through a Monte Carlo numerical simulation of (56). This results in the following estimate of the chaos coefficients:

$$
\boldsymbol{Y}_{\boldsymbol{\alpha}^{1} \cdots \boldsymbol{\alpha}^{p}}^{n_{s}}=\frac{1}{n_{s}} \sum_{k=1}^{n_{s}} \boldsymbol{Y}\left(a_{k}\right) \phi_{\boldsymbol{\alpha}^{1}}^{1}\left(\boldsymbol{X}^{1}\left(a_{k}\right)\right) \cdots \phi_{\boldsymbol{\alpha}^{p}}^{p}\left(\boldsymbol{X}^{p}\left(a_{k}\right)\right),
$$


where $\boldsymbol{Y}\left(a_{k}\right)$ and $\boldsymbol{X}^{j}\left(a_{k}\right)$ refer, respectively, to the $k$ th realization of $\boldsymbol{Y}$ and $\boldsymbol{X}^{j}$, where the former is obtained by solving the deterministic equation

$$
\boldsymbol{A}\left(\boldsymbol{X}^{1}\left(a_{k}\right), \ldots, \boldsymbol{X}^{p}\left(a_{k}\right)\right) \boldsymbol{Y}\left(a_{k}\right)=\boldsymbol{F} .
$$

The convergence of the chaos decomposition is analyzed by studying the convergence of the $\boldsymbol{r}$-sequence, $E\left\{\left\|\boldsymbol{Y}-\boldsymbol{Y}^{\boldsymbol{r}}\right\|_{\mathbb{C}}^{2}\right\}$. Although this sequence is indexed only by $\boldsymbol{r}$, the estimation of the chaos coefficients of $\boldsymbol{Y}^{\boldsymbol{r}}$ through sampling introduces a new parameter $n_{s}$. An appropriate metric for investigating the convergence of the overall approximation can thus be written as

$$
\mathcal{J}\left(\boldsymbol{r}, n_{s}\right)=\frac{1}{n_{s}} \sum_{k=1}^{n_{s}}\left\|\boldsymbol{Y}\left(a_{k}\right)-\boldsymbol{Y}^{\boldsymbol{r}, n_{s}}\left(a_{k}\right)\right\|_{\mathbb{C}}^{2}
$$

where

$$
\boldsymbol{Y}^{\boldsymbol{r}, n_{s}}\left(a_{k}\right)=\sum_{\boldsymbol{\alpha}^{1},\left|\boldsymbol{\alpha}^{1}\right| \leq r_{1}} \cdots \sum_{\boldsymbol{\alpha}^{p},\left|\boldsymbol{\alpha}^{p}\right| \leq r_{p}} \boldsymbol{Y}_{\boldsymbol{\alpha}^{1 \cdots} \boldsymbol{\alpha}^{p}}^{n_{s}} \phi_{\boldsymbol{\alpha}^{1}}^{1}\left(\boldsymbol{X}^{1}\left(a_{k}\right)\right) \times \cdots \times \phi_{\boldsymbol{\alpha}^{p}}^{p}\left(\boldsymbol{X}^{p}\left(a_{k}\right)\right) .
$$

6.3.2. Construction through Hilbertian projections and convergence analysis. An alternative construction [10] of the coefficients $\boldsymbol{Y}_{\boldsymbol{\alpha}^{1 \ldots} \boldsymbol{\alpha}^{p}}$ consists in substituting the truncated chaos decomposition of $\boldsymbol{Y}$, given in (59), into equation (56) and interpreting the resulting equality in the weak sense using the bilinear form in $\mathbb{H}^{(m)} \times \mathbb{H}$ defined in (21). This results in

$$
E\left\{\boldsymbol{A}\left(\boldsymbol{X}^{1}, \ldots, \boldsymbol{X}^{p}\right) \boldsymbol{Y}^{\boldsymbol{r}} \phi_{\boldsymbol{\alpha}^{1}}^{1}\left(\boldsymbol{X}^{1}\right) \cdots \phi_{\boldsymbol{\alpha}^{p}}^{p}\left(\boldsymbol{X}^{p}\right)\right\}=E\left\{\boldsymbol{F} \phi_{\boldsymbol{\alpha}^{1}}^{1}\left(\boldsymbol{X}^{1}\right) \times \cdots \times \phi_{\boldsymbol{\alpha}^{p}}^{p}\left(\boldsymbol{X}^{p}\right)\right\}
$$

for multi-indices $\boldsymbol{\alpha}^{k}$ such that $\left|\boldsymbol{\alpha}^{k}\right| \leq r_{k}$. Substituting (58) into the previous equation and assuming that (15) holds, and since $\boldsymbol{F}$ is a constant vector, yields

$$
\sum_{\boldsymbol{\alpha}^{1},\left|\boldsymbol{\alpha}^{1}\right| \leq r_{1}} \cdots \sum_{\boldsymbol{\alpha}^{p},\left|\boldsymbol{\alpha}^{p}\right| \leq r_{p}} \mathbb{A}_{\boldsymbol{\alpha}^{1 \ldots \boldsymbol{\alpha}^{p}} \boldsymbol{\beta}^{1 \ldots} \boldsymbol{\beta}^{p}} \boldsymbol{Y}_{\boldsymbol{\alpha}^{1} \ldots \boldsymbol{\alpha}^{p}}^{\boldsymbol{r}}=\boldsymbol{F} \delta_{\mathbf{0} \boldsymbol{\beta}^{1}} \cdots \delta_{\mathbf{0} \boldsymbol{\beta}^{p}},
$$

where deterministic matrix $\mathbb{A}$ is given by

$\mathbb{A}_{\boldsymbol{\alpha}^{1} \cdots \boldsymbol{\alpha}^{p} \boldsymbol{\beta}^{1} \ldots \boldsymbol{\beta}^{p}}=E\left\{\boldsymbol{A}\left(\boldsymbol{X}^{1}, \ldots, \boldsymbol{X}^{p}\right) \phi_{\boldsymbol{\alpha}^{1}}^{1}\left(\boldsymbol{X}^{1}\right) \cdots \phi_{\boldsymbol{\alpha}^{p}}^{p}\left(\boldsymbol{X}^{p}\right) \phi_{\boldsymbol{\beta}^{1}}^{1}\left(\boldsymbol{X}^{1}\right) \times \cdots \times \phi_{\boldsymbol{\beta}^{p}}^{p}\left(\boldsymbol{X}^{p}\right)\right\}$.

This finite-dimensional deterministic algebraic system of equations yields the chaos coefficients of $\boldsymbol{Y}^{r}$. Unlike the construction via sampling introduced previously, the computed chaos coefficients now depend on the multi-index $\boldsymbol{r}=\left(r_{1}, \ldots, r_{p}\right)$ used in the approximation. In a few special cases, the right-hand side of (67) can be evaluated analytically. In general, however, the mathematical expectation is estimated via Monte Carlo sampling resulting in matrix $\mathbb{A}_{\boldsymbol{\alpha}^{1} \ldots \boldsymbol{\alpha}^{p} \boldsymbol{\beta}^{1 \ldots} \boldsymbol{\beta}^{p}}^{n_{s}}$. The solution of (66) associated with this approximation is denoted by $\boldsymbol{Y}_{\boldsymbol{\alpha}^{1} \ldots \boldsymbol{\alpha}^{p}}^{n_{s}, \boldsymbol{r}}$. Since in the present case, realizations $\boldsymbol{Y}\left(a_{k}\right)$ of the exact solution are not available, estimation of the $\boldsymbol{r}$-sequence $E\left\{\left\|\boldsymbol{Y}-\boldsymbol{Y}^{\boldsymbol{r}}\right\|_{\mathbb{C}}^{2}\right\}$ cannot be directly investigated. Let a new $\boldsymbol{r}$-sequence be given by $E\left\{\left\|\boldsymbol{Y}^{\boldsymbol{r}}\right\|_{\mathbb{C}}^{2}\right\}$. If this sequence is upper bounded by a positive finite constant, then all of the chaos coefficients of $\boldsymbol{Y}^{\boldsymbol{r}}$ converge to the chaos coefficients of the exact solution. Indeed, the boundedness of the sequence $E\left\{\left\|\boldsymbol{Y}^{r}\right\|_{\mathbb{C}}^{2}\right\}$ implies the convergence of each chaos coefficient of $\boldsymbol{Y}^{\boldsymbol{r}}$ to the corresponding chaos coefficients of $\boldsymbol{Y}$. Thus, the sequence $E\left\{\left\|\boldsymbol{Y}^{r}\right\|_{\mathbb{C}}^{2}\right\}$ is a well-adapted sequence for monitoring the convergence of the chaos approximation. 
7. Conclusion. With the steady increase in availability and magnitude of computational resources, error analysis and fidelity in model-based predictions have taken a central role in modeling and computational sciences. Issues related to uncertainty quantification, both as far as modeling the uncertainty, propagating it through computational models, and managing its consequences are concerned, are gaining in significance. This paper has presented the mathematical foundation for formulating and resolving the technical issues associated with the probabilistic formulation of uncertainty quantification, using the polynomial chaos decomposition. The focus has been on the class of problems involving a finite number of basic random variables, with the Karhunen-Loeve decomposition permitting the efficient approximation of the infinite-dimensional case with a sequence of finite-dimensional subspaces.

The representations presented in the paper can be readily implemented into numerical codes, either using existing software via a Monte Carlo sampling scheme, or using stochastic codes that are adapted to the chaos decompositions. In either case, procedures for error analysis and convergence studies are presented in the paper. Such studies are essential for the meaningful combination of errors stemming from the discretization of the governing partial differential equations with those stemming from the stochastic discretization.

Finally, the methods presented herein can be extended in a straightforward manner to situations where the Hermitian range space of the transformations is replaced with more general spaces such as the space of matrices or a Banach space [20, 21].

8. Appendix. This appendix shows data needed to construct the most common orthogonal polynomials [1]. These in turn are used to construct the bases $\phi_{\ell}(q)$ of Hilbert spaces $\mathbb{H}_{j, k}$. Each of these polynomials is also associated with a onedimensional well-known probability density function, which is also indicated. Table 1 displays the polynomial name, its weight, support, associated probability measure, and the relation to the Hilbertian basis. Table 2 shows, for each polynomial, the recurrence relation allowing its construction. 
TABLE 1

Classical orthogonal polynomials, their weights, support, and normalization.

\begin{tabular}{|c|c|c|c|c|}
\hline Polynomial & Weight: $w(q)$ & Support: $s$ & $\begin{array}{l}\text { Associated proba- } \\
\text { bility measure }\end{array}$ & Hilbertian basis $\psi_{\ell}(q)$ \\
\hline$H_{\ell}(q):$ Hermite & $(2 \pi)^{-1 / 2} e^{-q^{2} / 2}$ & $\mathbb{R}$ & Gaussian & $H_{\ell}(q) / \sqrt{\ell !}$ \\
\hline$P_{\ell}(q):$ Legendre & 1 & ]$-1,1[$ & uniform & $P_{\ell}(q) \sqrt{\frac{2 \ell+1}{2}}$ \\
\hline$L_{\ell}^{a}(q):$ Laguerre & $\begin{array}{l}q^{a} e^{-q} \\
a>-1\end{array}$ & ] $0,+\infty[$ & Gamma & $L_{\ell}^{a}(q) \sqrt{\frac{\ell !}{\Gamma(a+\ell+1)}}$ \\
\hline$T_{\ell}(q)$ : Chebyshev & $\left(1-q^{2}\right)^{-1 / 2}$ & ]$-1,1[$ & Chebyshev & $T_{\ell}(q) \sqrt{\frac{2}{\pi\left(1+\delta_{0 \ell}\right)}}$ \\
\hline$P_{\ell}^{(a, b)}:$ Jacobi & $\begin{array}{l}(1-q)^{a}(1+q)^{b} \\
a>-1 ; b>-1\end{array}$ & ]$-1,1[$ & Beta & $P_{\ell}^{(a, b)}(q) \sqrt{\frac{\ell !(a+b+1+2 \ell) \Gamma(a+b+\ell+1)}{2^{a+b+1} \Gamma(a+\ell+1) \Gamma(b+\ell+1)}}$ \\
\hline
\end{tabular}


TABLE 2

Recurrence relations for the classical orthogonal polynomials.

\begin{tabular}{|l|c|c|c|}
\hline Polynomial & $\ell=0$ & $\ell=1$ & $\ell \geq 2$ \\
\hline$H_{\ell}(q)$ : Hermite & $H_{0}(q)=1$ & $H_{1}(q)=q$ & $H_{\ell}=q H_{\ell-1}-(\ell-1) H_{\ell-2}$ \\
\hline$P_{\ell}(q)$ : Legendre & $P_{0}(q)=1$ & $P_{1}(q)=q$ & $P_{\ell}=\frac{1}{\ell}(2 \ell-1) q P_{\ell-1}-\frac{1}{\ell}(\ell-1) P_{\ell-2}$ \\
\hline$L_{\ell}^{a}(q)$ : Laguerre & $L_{0}^{a}(q)=1$ & $L_{1}^{a}(q)=a+1-q$ & $L_{\ell}^{a}=\frac{1}{\ell}(2 \ell-1+a-q) L_{\ell-1}^{a}-\frac{1}{\ell}(\ell-1+a) L_{\ell-2}^{a}$ \\
\hline$T_{\ell}(q)$ : Chebyshev & $T_{0}(q)=1$ & $T_{1}(q)=q$ & $T_{\ell}=2 q T_{\ell-1}-T_{\ell-2}$ \\
\hline & & & $P_{\ell}^{(a, b)}=A_{\ell}(q) P_{\ell-1}^{(a, b)}-B_{\ell} P_{\ell-2}^{(a, b)}$ \\
$P_{\ell}^{(a, b)}:$ Jacobi & $P_{0}^{(a, b)}(q)=1$ & $P_{1}^{(a, b)}(q)=\frac{a-b}{2}+\left(\frac{a+b}{2}+1\right) q$ & $A_{\ell}(q)=\frac{(a+b+2 \ell-1)\left[(a+b+2 \ell-2)(a+b+2 \ell) q+a^{2}-b^{2}\right]}{2 \ell(a+b+\ell)(a+b+2 \ell-2)}$ \\
& & & $B_{\ell}=\frac{2(a+\ell-1)(b+\ell-1)(a+b+2 \ell)}{2 \ell(a+b+\ell)(a+b+2 \ell-2)}$ \\
\hline
\end{tabular}




\section{REFERENCES}

[1] M. Abramowitz and I. Stegun, Eds. (1972), Handbook of Mathematical Functions with Formulas, Graphs, and Mathematical Tables, U.S. Government Printing Office, Washington, DC.

[2] I. Babuska And P. Chatzipantelidis (2002), On solving elliptic stochastic partial differential equations, Computer Methods Appl. Mech. Engrg., 191, pp. 4093-4122.

[3] P. Billingsley (1995), Probability and Measure, Wiley and Sons, New York.

[4] B. Debusschere, H. Najm, A. Matta, O. Knio, R. Ghanem, and O. Le Maitre (2003), Protein labeling reactions in electrochemical microchannel flow: Numerical simulation and uncertainty propagation, Phys. Fluids, 15, pp. 2238-2250.

[5] R. Ghanem And A. Sarkar (2003), Reduced models for the medium-frequency dynamics of stochastic systems, J. Acoust. Soc. Amer., 113, pp. 834-846.

[6] R. Ghanem And M. Pellissetti (2002), Adaptive data refinement in the spectral stochastic finite element method, Comm. Numer. Methods Engrg., 18, pp. 141-151.

7] R. Ghanem And J. Red-Horse (1999), Propagation of uncertainty in complex physical systems using a stochastic finite element approach, Phys. D, 133, pp. 137-144.

[8] R. Ghanem And S. Dham (1998), Stochastic finite element analysis for multiphase flow in heterogeneous porous media, Transp. Porous Media, 32, pp. 239-262.

[9] R. Ghanem (1998), Scales of fluctuation and the propagation of uncertainty in random porous media, Water Resources Res., 34, pp. 2123-2136.

[10] R. Ghanem and P. Spanos (1991), Stochastic Finite Elements: A Spectral Approach, SpringerVerlag, New York.

[11] S. Janson (1997), Gaussian Hilbert Spaces, Cambridge University Press, Cambridge, UK.

[12] G. Kallianpur (1980), Stochastic Filtering Theory, Springer-Verlag, Berlin.

[13] A. Naylor and G. Sell (1982), Linear Operator Theory in Engineering and Science, SpringerVerlag, New York.

[14] P. Kree And C. Soize (1986), Mathematics of Random Phenomena, Reidel, Dordrecht, The Netherlands.

[15] S. KWApien And W. Woyczynski (1992), Random Series and Stochastic Integrals: Single and Multiple, Birkhäuser, Boston.

[16] O. Le Maitre, M. Reagan, H. Najm, R. Ghanem, and O. Knio (2002), A stochastic projection method for fluid flow. II. Random process, J. Comput. Phys., 181, pp. 9-44.

[17] M. Loeve (1977), Probability Theory, 4th ed., Springer-Verlag, New York.

[18] H. Matthies and C. Bucher (1999), Finite elements for stochastic media problems, Comput. Methods Appl. Mech. Engrg., 168, pp. 3-17.

[19] N. Oвата (1994), White Noise Calculus and Fock Space, Lecture Notes in Math. 1577, Springer-Verlag, Berlin.

[20] C. SoIze (2000), A nonparametric model of random uncertainties for reduced matrix models in structural dynamics, Probabilistic Engineering Mechanics, 15, pp. 277-294.

[21] C. Solze (2001), Maximum entropy approach for modeling random uncertainties in transient elastodynamics, J. Acoust. Soc. Amer., 109, pp. 1979-1996.

[22] N. Wiener (1938), The homogeneous chaos, Amer. J. Math., 60, pp. 897-936.

[23] D. Xiu, C.-H. Lucor, And G. E. Karniadakis (2002), Stochastic modeling of flow-structure interactions using generalized polynomial chaos, J. Fluids Engrg., 124, pp. 51-59. 